\title{
Estrategia de vigilancia para el control del tabaco en México: publicidad, promoción y patrocinio, empaque y etiquetado
}

\author{
Rosibel Rodríguez-Bolaños, MD, M ASS,(1) Luz Myriam Reynales-Shigematsu, MD, Dra en C,(1) \\ Norma A Ibañez-Hernández, Lic en Der, (2) René Santos-Luna, Ing Eléc,(3) Raydel Valdés-Salgado, MD, PhD (1) \\ Erika Ávila-Tang, MD, PhD, (4) Frances Stillman, MD, EdD.(4)
}

\begin{abstract}
Rodríguez-Bolaños R, Reynales-Shigematsu LM, Ibáñez-Hernández NA, Santos-Luna R, Valdés-Salgado R, Ávila-Tang E, Stillman F. Estrategia de vigilancia para el control del tabaco en México: publicidad, promoción y patrocinio, empaque y etiquetado. Salud Publica Mex 2010;52 supl 2:S254-S266.
\end{abstract}

\section{Resumen}

Objetivo. Describir las estrategias de publicidad, mercadeo, y venta productos del tabaco en 12 ciudades de México. Material y métodos. Los puntos de venta de tabaco, a $500 \mathrm{mts}$ alrededor de escuelas participantes de la Encuesta de Tabaquismo en Jóvenes (2005-2006), fueron identificados usando formatos observacionales y un Sistema de Información Geográfica en línea. Resultados. En promedio se encontraron 8 establecimientos y 5 puestos de calle alrededor de las 257 escuelas visitadas. $44.4 \%$ de los establecimientos tenía publicidad en interior, $8.3 \%$ poseían productos a la altura de los ojos de los niños y $6.5 \%$ tenía alguna promoción. El $33.6 \%$ tenía letrero de prohibición de venta a menores, en tanto que $44.4 \%$ de los establecimientos y $58.8 \%$ puestos de calle vendían cigarros sueltos. Conclusiones. Los productos de tabaco son ampliamente publicitados y comercializados alrededor de las escuelas. Existe un alto incumplimiento de las leyes referentes a la venta a menores y por menores. Es urgente implementar un sistema que permita una vigilancia continua de las estrategias de la industria y para el control del tabaco.

Palabras claves: tabaco; vigilancia epidemiológica; publicidad; etiquetado de productos; comercio de cigarros; México

\author{
Rodríguez-Bolaños R, Reynales-Shigematsu LM, \\ lbáñez-Hernández NA, Santos-Luna R, \\ Valdés-Salgado R, Ávila-Tang E, Stillman F. \\ Monitoring estrategy for control of tobacco in Mexico: \\ advertising, promotion and sponsorship, packaging and labeling. \\ Salud Publica Mex 2010;52 suppl 2:S254-S266.
}

\begin{abstract}
Objective. To describe strategies used in the publicity, marketing, and sale, of tobacco products in 12 cities in Mexico. Material and Methods. Tobacco products points of sale (POS) were identified within a $500 \mathrm{~m}$ radius of Global Youth Tobacco Survey (2005-2006) schools. We used observational surveys and an online Geographic Information System (GIS). Results. In the 257 schools visited, we found, on average, 8.3 stores and 5 street vendors around each of them. Forty-four percent of the stores had interior tobacco publicity, $8.3 \%$ had tobacco products at children's eye level, $6.5 \%$ had some promotion, $33.6 \%$ had a no selling to minors sign, and $44.4 \%$ of stores and $58.8 \%$ of street vendors sold single cigarettes. Conclusions. Tobacco products are largely publicized and marketed around schools. There is no compliance of tobacco control legislations in regards to selling to minors and single cigarettes. It is necessary to implement a surveillance system to monitor strategies for tobacco control and the tobacco industry.
\end{abstract}

Keywords: tobacco; epidemiological surveillance; publicity; products labeling; tobacco-derived products commerce; México

(I) Instituto Nacional de Salud Pública, Centro de Investigación en Salud Poblacional. Cuernavaca, Morelos, México

(2) Fundación Interamericana del Corazón. México

(3) Instituto Nacional de Salud Pública, Centro de Información para Decisiones en Salud Pública. Cuernavaca, Morelos, México

(4) Instituto Mundial para el Control del Tabaco. Universidad de Johns Hopkins. USA

Fecha de recibido: 26 de marzo de 2010 - Fecha de aceptado: 14 de mayo de 2010 Solicitud de sobretiros: Dra. Luz Myriam Reynales Shigematsu. Instituto Nacional de Salud Pública, Centro de Investigación en Salud Poblacional. Av. Universidad 655, col. Santa María Ahuacatitlán. 62100 Cuernavaca, Morelos, México. Correo electrónico: Ireynales@correo.insp.mx 
Es Convenio Marco de la Organización Mundial de la Salud para el Control del Tabaco (CMCT OMS), en su Artículo 20, insta a las Partes a establecer programas de vigilancia nacional, regional y mundial que permitan dar seguimiento a la epidemia del tabaquismo y sus determinantes así como a sus estrategias de control. ${ }^{1}$ La vigilancia se centra en el monitoreo, seguimiento y evaluación de las medidas de control del tabaco implementadas y en la identificación y caracterización de las tácticas de la industria tabacalera (IT); por lo tanto, es una actividad dinámica que da respuesta inmediata a las necesidades de información de las autoridades y tomadores de decisiones locales, regionales y mundiales. ${ }^{2}$

Con la implementación del CMCT OMS, -el cual México firmó (2003) y ratificó (2004)- se establecen las principales estrategias para controlar la epidemia del tabaquismo. En el Artículo 11 del CMCT OMS, se especifican las medidas relativas al empaquetado y etiquetado de los productos del tabaco. ${ }^{1}$ En las directrices para la aplicación de este artículo se establecen los requisitos eficaces en materia de empaquetado y etiquetado para comunicar los riesgos sanitarios y reducir el consumo de tabaco, así como prevenir las formas equívocas o engañosas. ${ }^{3}$

En el Artículo 13 del CMCT OMS se orienta a las Partes a introducir y hacer cumplir una prohibición total de toda forma de publicidad, promoción y patrocinio del tabaco. Si la Parte no está en condiciones de proceder a una prohibición total, aplicará restricciones de acuerdo con lo establecido en las directrices para la aplicación del Artículo 13. En las directrices se establecen las medidas eficaces sobre la prohibición de la publicidad directa o indirecta, la restricción sobre el uso de incentivos directos o indirectos que fomenten la compra, la prohibición de la publicidad promoción y el patrocinio en todos los medios de comunicación, incluyendo el internet, así como la publicidad, promoción y patrocinio transfronterizos. ${ }^{4}$

El control sobre la venta de productos a menores y por menores o sueltos, se describe en el Artículo 16; en él se especifican las medidas eficaces para prohibir la venta de productos de tabaco a los menores de edad, así como la prohibición de la distribución gratuita de productos de tabaco al público y especialmente a los menores, y la venta de cigarrillos sueltos o en paquetes pequeños que vuelvan más asequibles esos productos a este grupo poblacional. De igual manera, restringe el acceso directo a los productos de tabaco en los puntos de venta y la colocación de máquinas de autoservicio accesibles; además estipula la colocación de un anuncio claro y destacado de prohibición de venta a menores de edad.
En el Código Sanitario de los Estados Unidos Mexicanos de $1973^{5}$ ya se contemplaba el control sanitario del tabaco como materia de salubridad general. En 1984, se publicó la Ley General de Salud (LGS), ${ }^{6}$ y se estableció la obligatoriedad de que los productos contengan etiquetas en sus empaques que informen sobre la empresa que los produce, los ingredientes que contiene y en idioma español. ${ }^{7}$ En 1988 se publicó el Reglamento de la LGS, ${ }^{8}$ el cual incluyó declaraciones sobre contenidos de nicotina y alquitrán. Una reforma a la LGS en $1991,{ }^{9}$ incluyó advertencias escritas claras y visibles con tres mensajes rotatorios y alternantes cada 6 meses. La última reforma en $2004^{10}$ contempló que el tamaño de la advertencia debería ser equivalente a 25\% de cualquiera de las caras (frontal o trasera), en adición a una leyenda en una de las caras laterales, con mensajes para orientar al fumador hacia los programas de tratamiento para dejar de fumar. La legislación vigente se encuentra establecida en los Artículos 18 a 22 de la Ley General para el Control del Tabaco (LGCT) publicada en el 2008. ${ }^{11}$

En esta última reforma en la LGS se prohibió totalmente la publicidad en radio, televisión e internet, situar los anuncios a menos de 200 metros de escuelas, hospitales, parques y clubes deportivos, educativos o familiares, que los anuncios exteriores rebasaran el tamaño de 35 metros cuadrados y su aparición en las revistas dirigidas a niños y adolescentes o aquellas con contenidos educativos, deportivos o de salud. Se prohibió en farmacias, boticas, hospitales y centros de salud. La legislación vigente se encuentra establecida en los artículos 23 a 25 de la LGCT.

Desde 1984 la LGS, en su Artículo 277, establecía expresamente que en ningún caso y de ninguna forma se podrá expender tabaco a menores de edad. En el Reglamento de Control Sanitario de Productos y Servicios de 1999 se estableció que no podría vender cigarros o cigarrillos por unidad ni en envases menores de 14 unidades. Obligaba a los establecimientos a exhibir los productos de manera que no fueran de libre acceso al público y exigir una identificación oficial cuando la apariencia física del comprador no demostrara su mayoría de edad. Actualmente la legislación vigente se encuentra en los artículos 15 a 17 de la LGCT.

La IT ha buscado estrategias de publicidad, promoción y patrocinio de los productos de tabaco para llegar a los consumidores y, en especial, a los grupos más vulnerables: los jóvenes y las mujeres. Los puntos de venta han demostrado ser el lugar ideal para exponer anuncios de propagandas y promoción, en donde los niños y adolescentes acuden con frecuencia. ${ }^{12}$ Esta publicidad generalmente ocupa un lugar destacado y visible $^{13,14}$ y está diseñada para posicionar sus marcas 
en los niños e incitar a los fumadores a comprar sus productos compulsivamente. ${ }^{15}$

En el Instituto para el Control Global del Tabaco (IGTC), de la Universidad Johns Hopkins, han desarrollado una metodología de vigilancia para el control del tabaco (Tobacco Control Tracking Tools) ${ }^{2}$ con el objetivo de fortalecer la capacidad de los países en la vigilancia de las tácticas de la industria tabacalera y de seguimiento y evaluación de las medidas de control del tabaco. Esta metodología ha sido implementada de manera exitosa en varios países del mundo, incluyendo a México, donde esta herramienta se ha adaptado a un Sistema de Información Geográfica (SIG) en línea que permite el manejo de la información a nivel local y su disponibilidad a nivel central. ${ }^{16}$

En este manuscrito los autores tienen como objetivo identificar, caracterizar y vigilar las estrategias de publicidad, mercadeo, venta, empaque y etiquetado de los productos del tabaco en 12 ciudades de México, a partir de metodologías de vigilancia al utilizar un Sistema de Información Geográfica.

\section{Material y métodos}

\section{Fuentes de información}

Se obtuvo información mediante un sistema de vigilancia centinela de la publicidad, promoción y patrocinio, empaque y etiquetado de los productos de tabaco en México y por medio de dos fuentes de información y una herramienta tecnológica:

a) La Encuesta de Tabaquismo en Jóvenes (ETJ), ${ }^{17}$ pertenece al Sistema de Vigilancia Epidemiológica Global del Tabaco (GTSS, por sus siglas en inglés) y ha sido diseñada para la vigilancia mundial del consumo de tabaco entre los adolescentes. En México, esta encuesta se ha realizado en estudiantes entre 12 a 16 años de edad, en escuelas secundarias públicas y privadas del área urbana. La muestra de escuelas seleccionadas en los años 2005 y 2006 en las ciudades de Ciudad Juárez, Cuernavaca, Culiacán, Chetumal, Distrito Federal, Guadalajara, Nuevo Laredo, Puebla, Tapachula, Tepic, Tijuana y Toluca sirvieron de referencia para implementar la estrategia de vigilancia.

b) Tobacco Control Tracking Tools, este instrumento de información utiliza cuatro formatos observacionales, para vigilancia de: a) establecimientos, b) puestos de calle, c) empaquetado y etiquetado, $y$, d) filantropía. Donde contienen indicadores específicos que permiten identificar y caracterizar las tácticas de la IT y evaluar el cumplimiento de las medidas de control relativas a:

- Publicidad y promoción. Permite identificar y caracterizar toda forma de comunicación, recomendación o acción comercial para promover directa o indirectamente un producto o el uso de tabaco.

- Venta de cigarros a menores y por menores. Permite identificar el acceso de los jóvenes a los productos de tabaco y vigilar el cumplimiento de la legislación en cuanto a la prohibición de venta a los menores de la edad, la prohibición de la fabricación de objetos que puedan resultar atractivos para los menores, y el acceso de los menores a las máquinas expendedoras de tabaco.

- Patrocinio. Identifica toda forma de contribución o cualquier acto, actividad o individuo con el fin de promover directa o indirectamente un producto de tabaco o el uso de tabaco.

- Empaquetado y etiquetado de cajetillas de cigarrillos. Permite vigilar el cumplimiento de la legislación referente a empaque y etiquetado; advertencias (color, texto y porcentaje que abarca en la etiqueta), contenido de los mensajes, línea telefónica de ayuda para la cesación, información sobre ingredientes y emisiones y procedencia del producto.

c) Sistema de Información Geográfica (SIG) para la vigilancia de las estrategias de control del tabaco, es un sistema WEB integrado por cuatro módulos. El primer módulo es un subsistema para la captura y almacenamiento de la información de los cuatro formatos que caracterizan la publicidad en los puntos de venta (establecimientos y puestos de calle), la venta de cigarrillos a menores y al menudeo, espectaculares y patrocinio, empaque y etiquetado de las cajetillas. El segundo permite georreferenciar las escuelas y determinar el espacio de vigilancia mediante la delimitación de áreas concéntricas alrededor de cada punto (buffers). ${ }^{18} \mathrm{El}$ tercero permite adicionar evidencias fotográficas y relacionarlas con las escuelas y los puntos de venta georreferenciados. El cuarto se refiere a la presentación de los indicadores de resultados (figura 1).

\section{Estrategia de campo}

El proyecto Tobacco Control Tracking Tools y el SIG fue coordinado por el Instituto Nacional de Salud Pública de México (INSP), el cual cuenta con el apoyo del Ins- 


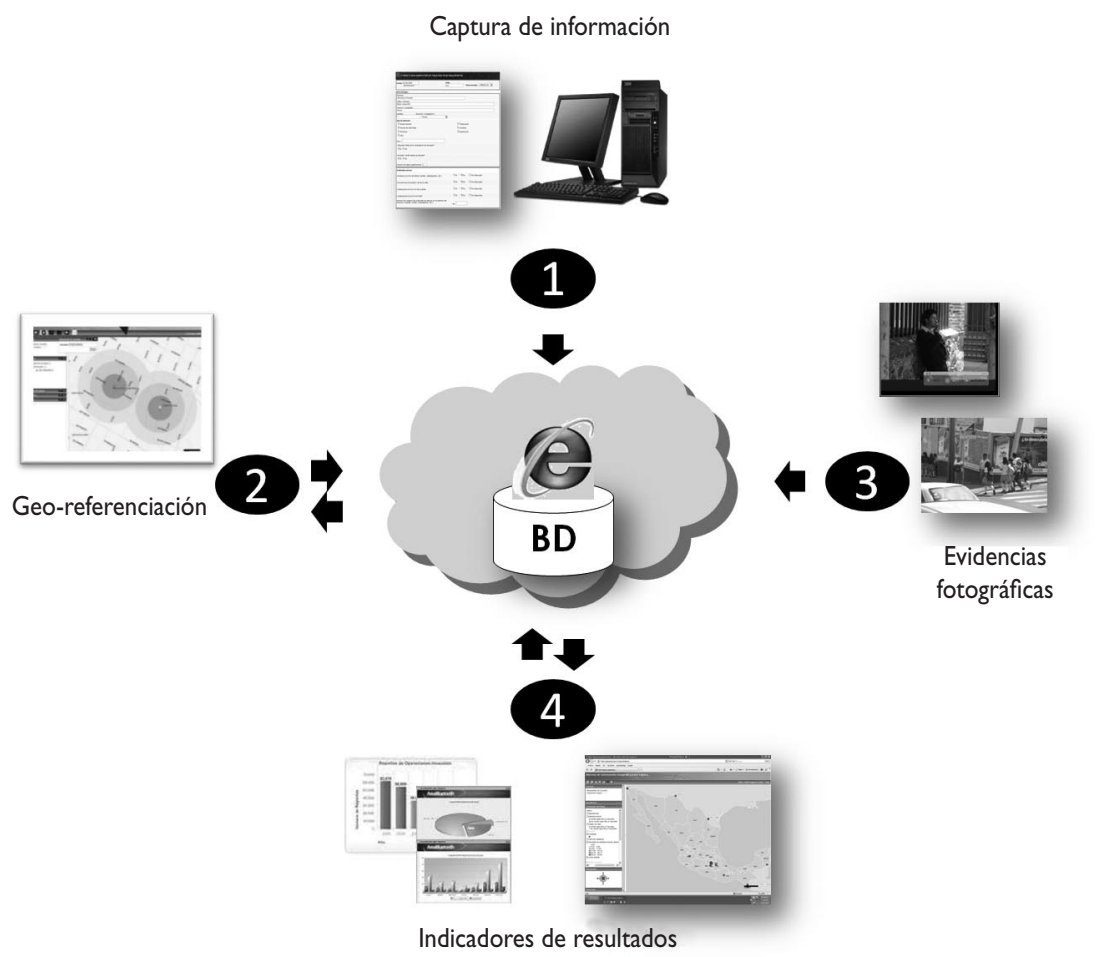

Figura I. Modelo conceptual del Sistema de Información Geográfica Web. 2008

tituto Global para el Control del Tabaco de la Universidad Johns Hopkins. Esta investigación fue aprobada por el Comité de Ética del INSP. El levantamiento de la información se realizó de manera simultánea en las 12 ciudades entre abril y junio de 2008 y estuvo a cargo de los Consejos Estatales Contra las Adicciones (CECAS). Previo al levantamiento se realizó un taller (teórico-práctico) que permitió la capacitación de los observadores en la metodología de vigilancia y estandarizarlos en lo referente a las definiciones, utilización de formatos y captura de la información en el SIG. El proceso de estandarización se enfocó en las siguientes unidades de observación:

a) Puntos de referencia. Muestra de escuelas participantes en la ETJ 2005-2006. Las escuelas fueron georreferenciadas previamente, lo que permitió contar con los mapas que identificaban las zonas de vigilancia de $200 \mathrm{~m}, 300 \mathrm{~m}$ hasta $500 \mathrm{~m}$ alrededor de las escuelas. En campo se observaban los accesos como calles, avenidas principales, puentes peatonales, entre otros, y la ubicación de los puntos de venta. b) Puntos de venta. Los puntos de venta fueron clasificados en establecimientos y puestos de calle; ambos fueron georreferenciados y relacionados al punto de referencia (escuela).

En establecimientos:

- Publicidad directa. Interna: anuncios como póster, carteles, etc., iluminados, con movimientos. Externa: anuncios de cualquier tipo en las puertas y ventanas que se puedan ver de la calle.

- Publicidad indirecta. Ubicación de los estantes y de productos a la altura de los ojos de los niños, así como objetos de la IT que tuvieran alguna utilidad como espejos, ceniceros, entre otros. Paredes o anaqueles pintados con colores o emblemas, ropa o artículos personales con colores o emblemas.

- Promoción. Precio especial o con promocional (regalo) y ofertas o promociones con cupones.

- Precio. Se registró el precio ( por cigarro suelto, cajetilla y si se vendía por paquete) de la marca de cigarro Marlboro, por ser la marca más vendida en el mundo y en el país, y una marca 
de cigarro local. Se observó la presencia del letrero de prohibición de venta de cigarrillo a menores de edad.

En puestos de calle:

- Se identificaban todas las formas de publicidad, promoción y venta de cigarros a menores y por menores que se observaba en los establecimientos.

c) Empaque y etiquetado de los productos de tabaco. Se obtenía una muestra de una cajetilla o cigarros sueltos por escuelas de establecimientos y puestos de calle. Se caracterizó tamaño de advertencia ( $\%$ aproximado en relación con el área donde se ubica), colocación y frase de advertencia, si contaban con estampillas de impuestos (importados), reporte sobre contenidos y mensaje sobre línea telefónica de ayuda para dejar de fumar. Se tomó como referencia la cajetilla de Marlboro rojo por ser la marca de mayor consumo; además se obtuvieron productos de las marcas más populares y de los que tenían un empaque novedoso o tenían algún tipo de promoción.

\section{Integración y análisis de la información}

Los formatos y la georreferenciación fueron capturados en el SIG a nivel local por los coordinadores estatales de los CECAS. El proceso de validación de los datos fue realizado en el INSP, cotejando la información de los formatos en el SIG. De igual manera, las evidencias fotográficas fueron registradas con el código de cada escuela para su identificación y fueron almacenadas en la plataforma del sistema. El análisis de la información se realizó utilizando el paquete estadístico STATA versión 10.1.

\section{Resultados}

En las 12 ciudades seleccionadas, se visitaron 257 escuelas y se encontró en el área de influencia un total de 2580 puntos de ventas, de los cuales 2128 eran establecimientos y 452 puestos de calle. En promedio, alrededor de cada escuela se identificaron ocho establecimientos y cinco puestos de calle. Las ciudades con mayor densidad de establecimientos (más de 15 establecimientos) alrededor de cada escuela fueron Chetumal, Toluca y la Ciudad de México. En 13.9\% de las escuelas los establecimientos eran visibles desde su puerta principal.

El cuadro I presenta los hallazgos principales relacionados con la publicidad, promoción, venta a menores y por menores en establecimientos y puestos de calle en las ciudades seleccionadas.

\section{Publicidad}

En general, la publicidad interior estuvo presente en $37.6 \%$ de los establecimientos, con más de $70 \%$ en las ciudades de Culiacán y Guadalajara. En promedio, un porcentaje bajo de establecimientos exhibía publicidad exterior (5.3\%), sin embargo, en Guadalajara se observó en $18 \%$ de los establecimientos. Llama la atención que en la Ciudad de México no se encontró ningún establecimiento con publicidad exterior (cuadro I).

En cuanto a la publicidad indirecta localizada en el interior de los establecimientos (estantería de la IT cerca de las cajas registradoras y productos colocados a la altura de los ojos de los niños), fue proporcionalmente menor en las ciudades fronterizas del sur (Tapachula y Chetumal) y del norte (Nuevo Laredo, Ciudad Juárez y Tijuana), comparado con las ciudades de Occidente (Guadalajara, Tepic) y del centro (Toluca, Ciudad de México, Cuernavaca y Puebla), donde la publicidad fue mayor que en el resto de las ciudades. En un análisis espacial de los datos de la prevalencia de consumo de tabaco estimada en la ETJ y los datos de publicidad de productos de tabaco, se observa que las ciudades con mayor publicidad en los establecimientos tienen mayor prevalencia de fumadores actuales, contrariamente a aquellas con menor publicidad, que tienen menor prevalencia (figura 2).

La publicidad en los puestos de calle fue casi nula. Aunque no se encontraron puestos de calle en las ciudades de Nuevo Laredo y Tapachula, en esta última se observaron vendedores ambulantes llamados "canguros", los cuales ofrecen cigarrillos sueltos y dulces. ${ }^{19}$

\section{Promoción}

En general, 6.5\% de los establecimientos ofrecían precios especiales o algún tipo de promoción por la compra. Las ciudades donde se observó más promoción fueron Tepic y Toluca donde 12 y $14 \%$ de los establecimientos, respectivamente, ofrecía precios especiales (cuadro I).

La mayor proporción de establecimientos que ofrecía algún tipo de regalo por la compra de productos de tabaco se localizaban en las ciudades fronterizas de Tijuana y Ciudad Juárez (7.8 y 5.3\% de los establecimientos, respectivamente) y en las ciudades del centro Cuernavaca y Toluca (6.9 y $6.3 \%$ de los establecimientos respectivamente). Hay que resaltar que en Puebla, Chetumal y Culiacán no se encontró ningún tipo de promoción. 


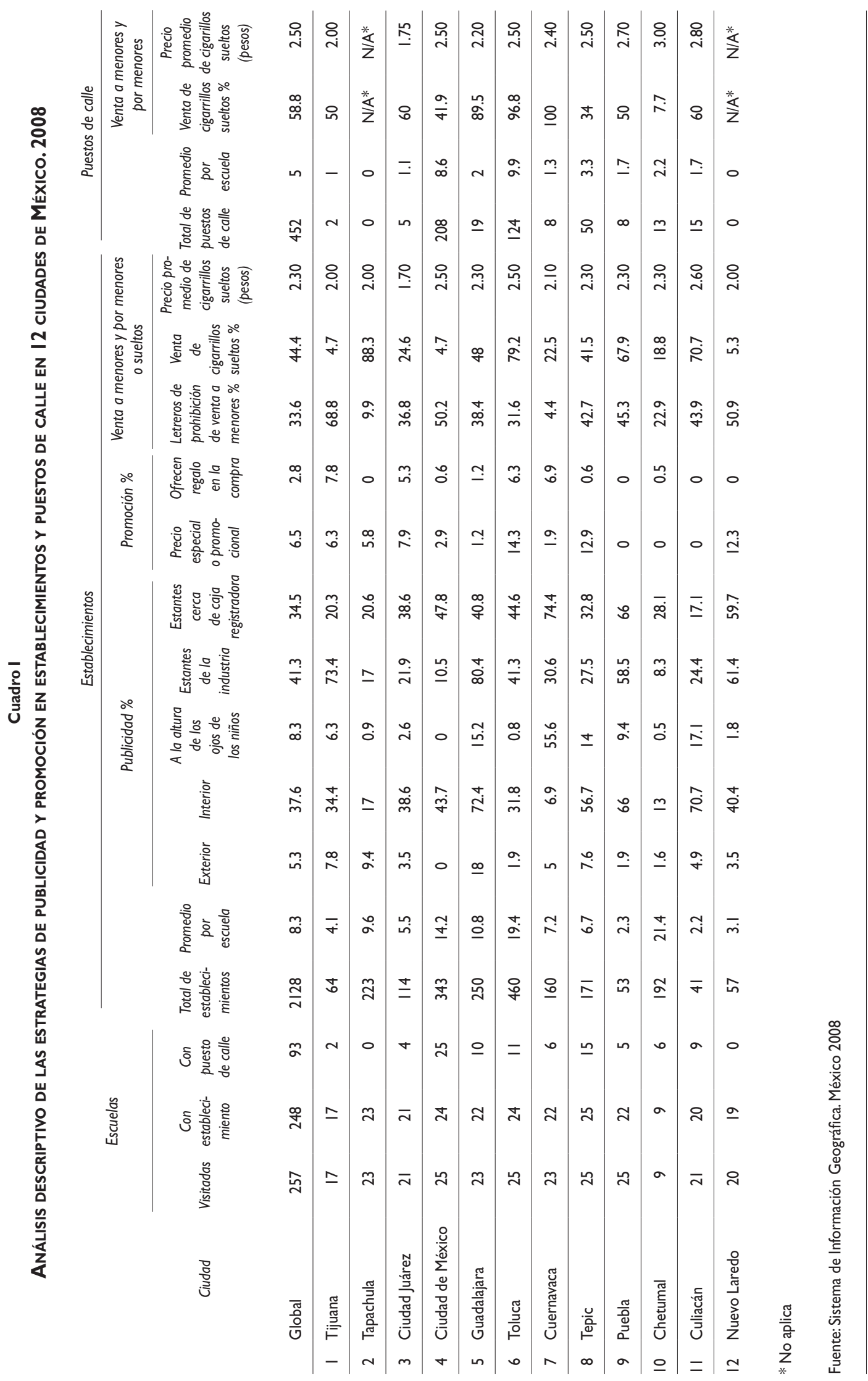




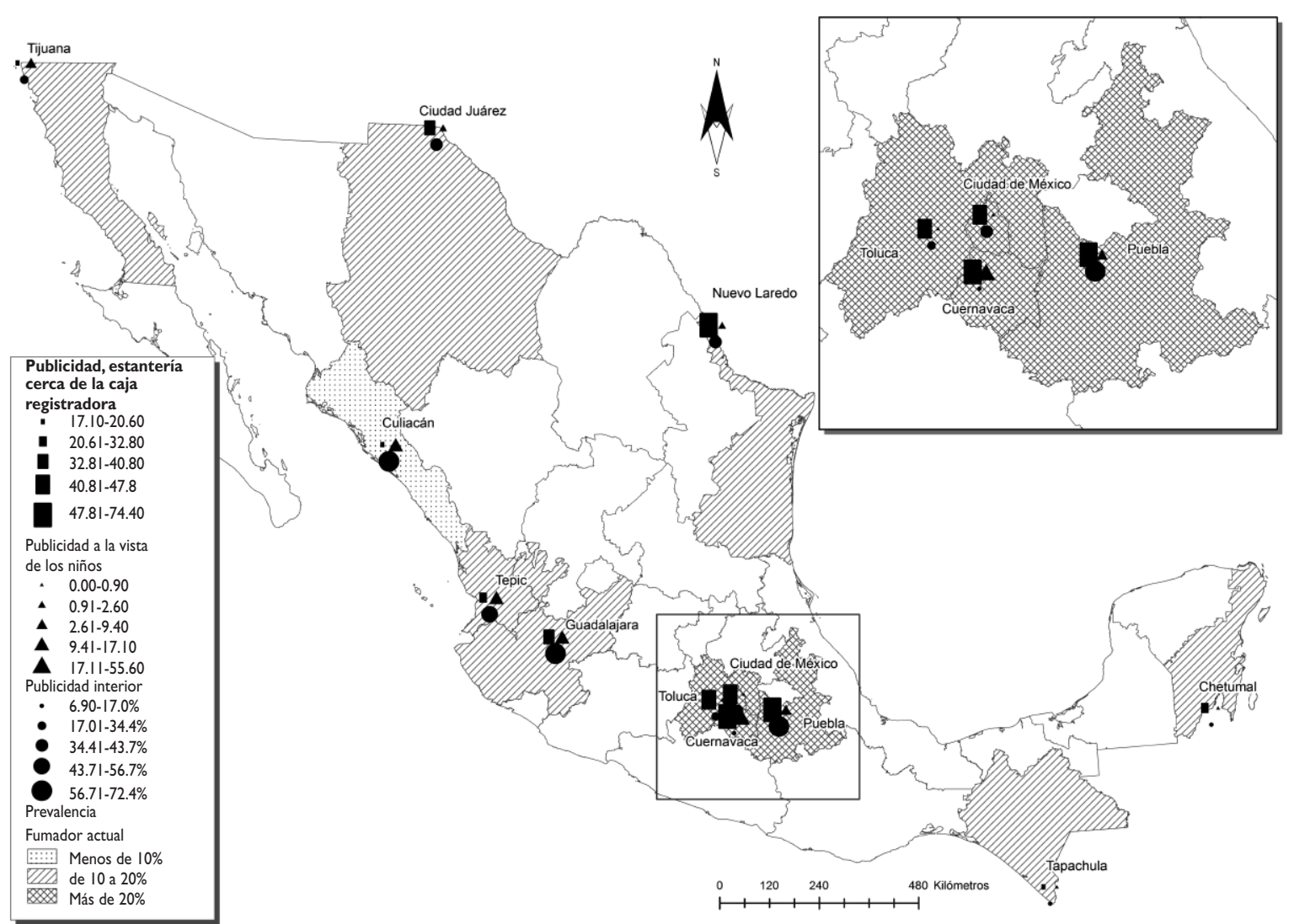

Figura 2. Prevalencia de jóvenes fumadores y publicidad de productos del tabaco en el interior de LOS ESTABLeCImientos en 12 ciudades de México. Sistema de informacióno geográfica WeB. 2008

En los puestos de calle no se observó ningún tipo de promoción, a excepción de uno, en la ciudad de Toluca, exclusivo para la venta de productos de tabaco, que exhibía publicidad y ofrecía promocionales de regalo por la compra del producto. ${ }^{19}$

\section{Venta de cigarros a menores y por menores (sueltos)}

Los observadores pudieron comprar cigarros sueltos en $44.4 \%$ de los establecimientos y en casi $60 \%$ de los puestos de calle. La evidencia fotográfica demuestra que los jóvenes tienen acceso a los cigarros sueltos tanto en los establecimientos como en los puestos de la calle. El precio promedio de venta de los cigarros sueltos fue de 2.30 y 2.50 pesos en los establecimientos y puestos de calle respectivamente. En establecimientos de Cuernavaca y Tepic se encontraron máquinas de autoservicio vendedora de cigarros sueltos localizada en un lugar visible y accesible al público. ${ }^{19}$
Un análisis espacial de la ciudad de Toluca permite visualizar el número de establecimientos y puestos de calle que venden cigarros a menudeo en la zona de influencia (500 metros) alrededor de las escuelas, donde $79.2 \%$ de los establecimientos y $97 \%$ de los puestos de calle venden cigarros de manera ilegal (figura 3).

En general, en 33.6\% de los establecimientos se observó el letrero con la prohibición de venta de cigarro a menores de edad y algunos se localizaban cerca de anuncios publicitarios. Estos letreros son proporcionados por la IT por lo que muestran el logo de la industria. ${ }^{19}$

\section{Espectaculares y patrocinio de eventos}

Cuernavaca fue la única ciudad donde se encontraron, alrededor de dos escuelas, espectaculares promocionando una marca de cigarro, sin que se localizaran a menos de 200 metros de la escuela. Durante el periodo del levantamiento de campo se promocionaron ampliamente los conciertos de Marlboro MX Beat en las 


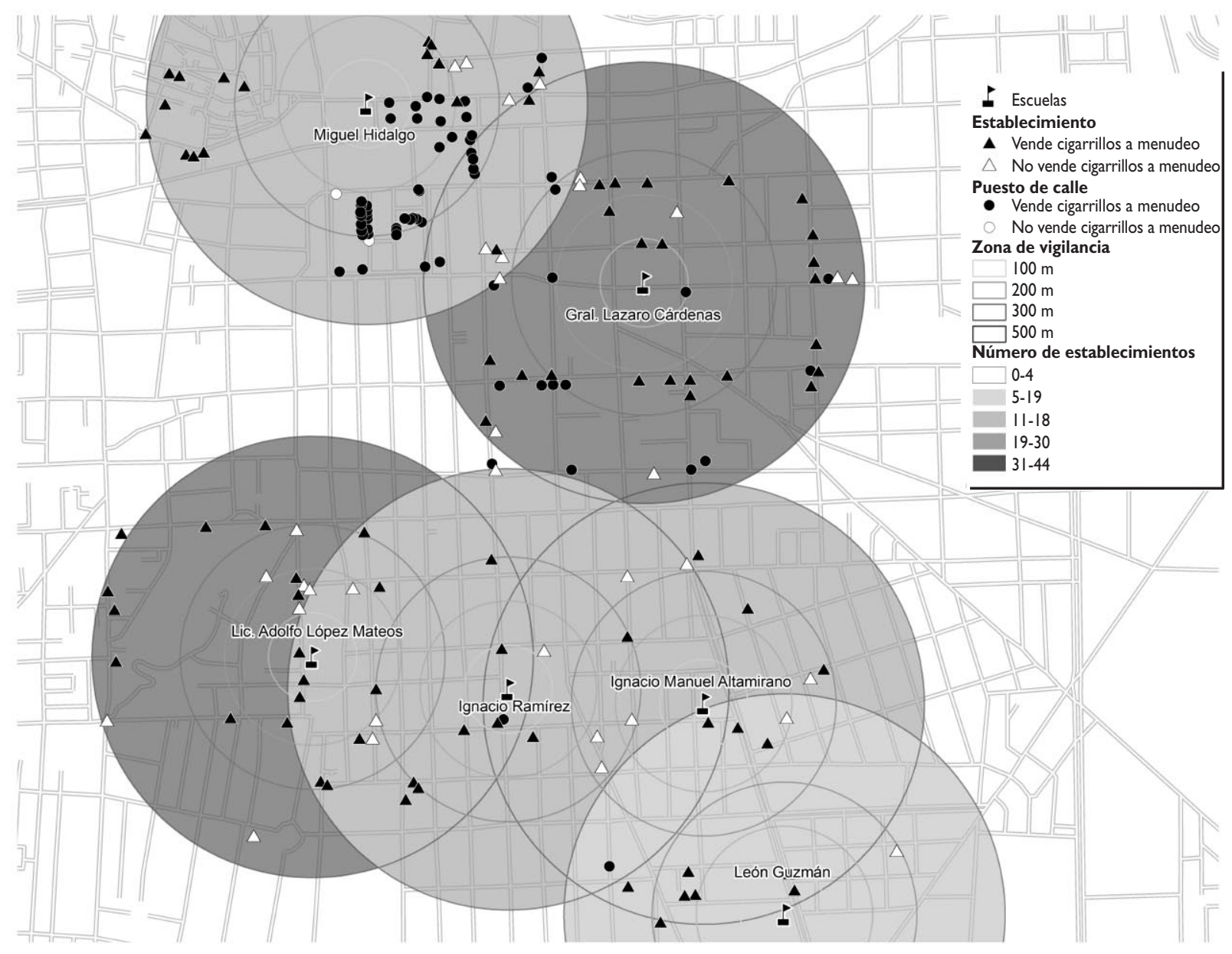

Figura 3. Número de establecimientos y puestos de CAlle QUe venden cigarros a menudeo (sueltos). Escuelas de la CIUDAd de TOluCA. Sistema de INFormación geográfica WEB. 2008

ciudades de Guadalajara, Puebla y Ciudad de México; ${ }^{20}$ sin embargo, en las áreas de influencia alrededor de las escuelas seleccionadas no se observó ningún tipo de publicidad de éste $u$ otro evento patrocinado por la industria tabacalera.

\section{Empaquetado y etiquetado}

Se obtuvieron un total de 245 cajetillas de cigarros. 89\% contenían 20 unidades (rango 14-25). La marca con mayor número de presentaciones fue la familia Marlboro en cajetilla dura o blanda y en versiones (rojo y azul); saborizados (menthol -verde-, mild flavor) e indicativos como Lights (blanco), suaves, wides y after dark. En otras marcas también se encontraron frases en inglés como smooth, natural flavor y generous flavour que corresponden a la marca Camel. Los diseños de las cajetillas de esta marca son las más atractivas y proporcionan regalos por la comprar de sus productos. Los superslims de Benson \& Hedges son cigarros delgados orientados a la población femenina.

Dentro de las marcas nacionales destacan Faros y Alas, los cuales se encontraron en todas las ciudades; algunos de sus productos tenían promociones. La marca con mayor número de presentaciones fue Delicados de la que se encontraron cuatro presentaciones diferentes; otras marcas poco conocidas fueron Picudo (manufacturada en Quintana Roo), que se comercializa principalmente en las ciudades del centro, y la marca Posse (manufacturados en Tamaulipas), que se encontró en Guadalajara.

En $92.5 \%$ de las cajetillas se encontraron leyendas de advertencias ubicadas en la cara posterior. La etiqueta (incluyendo el borde) abarcaban de 34 a 54\% de 
la superficie total; sin embargo, el texto en la etiqueta cubría de 14 a $42 \%$ aproximadamente. Los textos están escritos en mayúsculas y el tipo de letra corresponde a Arial Narrow. El color de las letras, en general, fue negro, sin embargo, hay variación de acuerdo con el color del empaque. En cuanto a los contenidos de los mensajes se encontraron los tres vigentes, y se utilizan alternadamente: "Dejar de fumar reduce importantes riesgos en la salud", "Fumar es causa de cáncer y enfisema pulmonar", "Fumar durante el embarazo aumenta el riesgo de parto prematuro y de bajo peso en el recién nacido". El mensaje "El tabaco es nocivo para la salud" se encontró en una cajetilla procedente de Colombia.

El 4.4\% no tenían la leyenda "Venta prohibida a menores" y el mensaje de la línea de ayuda para dejar de fumar fue agregado en un prospecto interno $(10.4 \%)$ y externo (4.4\%) del empaque. El 16.4\% fueron importados y $7.4 \%$ venían en otro idioma (inglés), los cuales se presumen que son de contrabando. En la actualidad no hay forma de identificar cuando un producto es de contrabando ya que no cuentan con marbete o la declaratoria "Para venta exclusiva en México". El 7.4\% de las cajetillas no menciona el contenido de alquitrán, nicotina y monóxido de carbono. La fecha de caducidad estaba inscrita en $86.5 \%$ de las cajetillas.

\section{Discusión}

La publicidad y promoción de los productos del tabaco está dirigida principalmente a los jóvenes. Los puntos de venta, tanto los establecimientos como los puestos de calle, permiten el acceso de manera fácil, promoviendo el inicio de su consumo. Aun con la existencia de evidencia que corrobora este hecho, los jóvenes del mundo, incluyendo los mexicanos, continúan expuestos sin reservas a este tipo de publicidad. 19,21,22

Estudios epidemiológicos, incluyendo un meta-análisis que utiliza metodologías similares, correlacionando información proveniente de encuestas de tabaquismo en escuelas y registros de establecimientos que venden y publicitan productos del tabaco, demuestran consistentemente que los adolescentes son vulnerables a la publicidad y promoción de los productos del tabaco y que estas tácticas hacen que los no fumadores sean más susceptibles a probar los cigarrillos y convertirse en fumadores. ${ }^{23-24}$ Estos estudios han encontrado asociación entre el número de establecimientos con publicidad del tabaco con el inicio del consumo (experimentación) y el consumo establecido de tabaco. ${ }^{25}$ Se concluye que la publicidad modifica el rumbo de la conducta de los jóvenes hacia el consumo de tabaco.
La ETJ a nivel mundial estima que alrededor de $70 \%$ de los jóvenes ha estado expuestos a publicidad en revistas y periódicos y más de $80 \%$ ha visto espectaculares promoviendo productos del tabaco, estrategias que fomentan actitudes positivas en los jóvenes y los motivan a fumar. ${ }^{26}$ De acuerdo con la ETJ en México, las ciudades del centro del país (Toluca, Ciudad de México y Puebla) reportan las mayores prevalencias de tabaquismo en los adolescentes; coincidentemente, fueron las que en este estudio reportaron mayor proporción de publicidad indirecta en el interior de los establecimientos. La ETJ refiere que cerca de $20 \%$ de los jóvenes de Puebla y Ciudad de México tienen algún artículo con el logo de una marca de tabaco. ${ }^{27,28}$ En Toluca, $13.2 \%$ de los jóvenes ha recibido cigarro gratis por un representante de la industria del tabaco y más de $40 \%$ de los jóvenes fumadores reporta que no se les negó la venta de cigarros a pesar de su edad. Esta información es consistente con los hallazgos del presente estudio donde sólo $31.6 \%$ de los establecimientos ostenta el letrero de prohibición de venta a menores y cerca de $80 \%$ vende cigarros sueltos (cuadro II).

Se realizaron dos estudios en la Ciudad de México (1999 y 2002) con el objetivo de identificar la venta de cigarros a menores. Los resultados obtenidos muestran en la primera medición que en $79 \%$ de las tiendas visitadas se les vendió cigarros a menores, y sólo 12\% de las tiendas tenían letreros indicando la prohibición de venta a menores de edad. ${ }^{29}$ En la segunda medición, 73\% de las tiendas le vendieron cigarros a menores de edad y en $21 \%$ se encontraron carteles de advertencia de "no venta a menores". ${ }^{30}$ En este último estudio reportan que en $94 \%$ de los puestos de calle se les vendió cigarros a menores de edad. En Ciudad Juárez encontraron que en $98 \%$ de los establecimientos se les vendió cigarros a los menores y en las tiendas visitadas no se encontró ningún cartel de advertencia. ${ }^{31}$ La venta de cigarros es mayor a lo encontrado en la ETJ y la colocación de letreros de prohibición es menor a lo reportado por el sistema en esas ciudades, lo que podría explicarse por las medidas más restrictivas que se han impulsado con la firma y ratificación del CMCT OMS.

Varios estudios han demostrado que la publicidad de los productos de tabaco utiliza imágenes que provocan deseo de fumar. ${ }^{32,33}$ Por esta razón, en el actual Reglamento de la LGCT se establece que la información sobre los productos del tabaco que se exhiba en el interior de los puntos de venta deberá ser igual para todos los productos equivalentes, y consistirá sólo en el nombre y precio de los mismos. La colocación de los productos del tabaco junto con productos de mayor consumo entre los niños y adolescentes (por ejemplo 


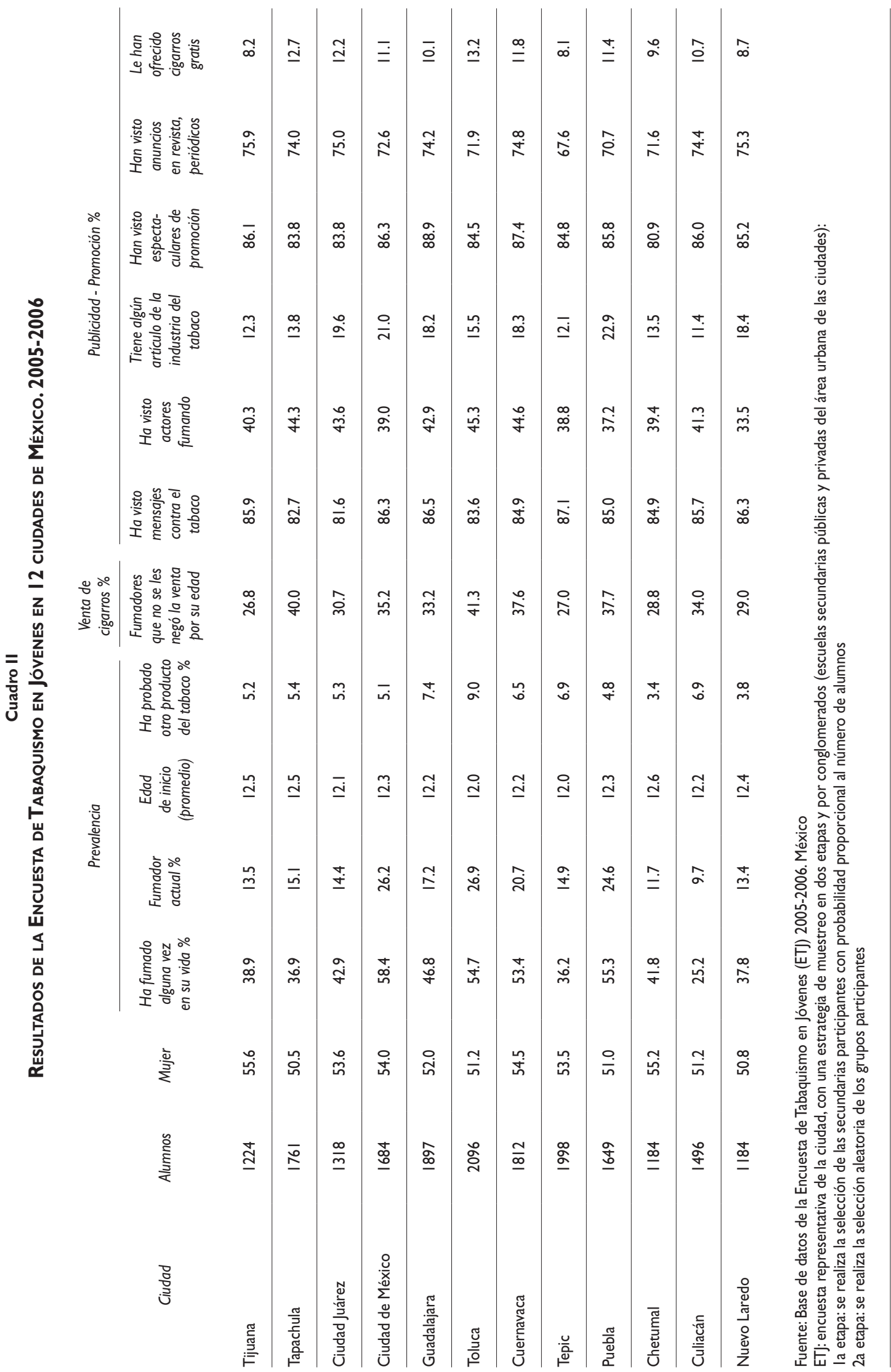


los dulces y chocolates) es una estrategia de la IT para posicionar sus marcas en la mente de los adolescentes del futuro. ${ }^{34}$ Esta fue una estrategia común observada en todas las ciudades estudiadas no sólo en los establecimientos sino en los puestos de calle. ${ }^{16,19} \mathrm{Si}$ bien el porcentaje de estantes con logotipos de la industria no fue tan alto como en otros estudios, la ubicación de ellos es estratégica pues el hecho de estar colocados próximos a las cajas registradoras hace que todos los compradores estén expuestos a la publicidad. La ciudad de Cuernavaca fue la única donde se encontraron espectaculares alrededor de dos escuelas. Esta evidencia sugiere claramente incumplimiento de la ley.

Este estudio demuestra una vez más que en México no se cumple la legislación en cuanto a la prohibición de venta a menores y al menudeo. La evidencia fotográfica comprueba la venta de cigarros sueltos a los menores y el libre acceso a máquinas de autoservicio de cigarros al menudeo en los establecimientos. ${ }^{19,30-32}$ De igual manera, es importante destacar que en 6 de cada 10 puestos de la calle alrededor de las escuelas se venden cigarros al menudeo, los puestos de calle son generalmente informales y móviles (algunos sólo se colocan a las horas de entrada y salida de las escuelas) lo que hace más complejo la vigilancia y cumplimiento. En este punto en particular vale la pena rescatar el mecanismo de denuncia ciudadana que introduce la LGCT, una herramienta para la sociedad civil que apoya el cumplimiento de la ley. En estudios revisados no se encontraron datos sobre venta en puestos ambulatorios pero sí del nivel de publicidad en ellos. 35,36

$\mathrm{Si}$ bien es cierto, se exige a los establecimientos colocar un letrero de prohibición de venta a menores; éstos son proporcionados por la IT y muestran el logotipo de la misma. En el CMCT OMS se considera la "extensión de marca y uso de marca común" cuando se utiliza un nombre, un emblema, una marca comercial, un logotipo, una insignia comercial o cualquier otro rasgo distintivo de un producto, por lo que representa un tipo de publicidad indirecta. No es coincidencia que en varios establecimientos visitados en este estudio este letrero estaba colocado justo al lado de la publicidad de los productos de tabaco. Con la publicación de la LGCT y su Reglamento, se contempla que la SSA es la responsable de emitir las disposiciones para la colocación y contenido de los letreros que se ubicarán en lugares donde haya venta de productos del tabaco; de igual forma, se establece que los exhibidores de los productos de tabaco que se encuentren en los establecimientos que comercialicen, vendan, distribuyan, suministren o expendan productos del tabaco deberán incluir las leyendas "Venta prohibida de cigarrillos por unidad" y "Se prohíbe el comercio, venta, distribución o suministro a menores de edad".
Otro punto que es importante mencionar es que los propios empaques de los productos de tabaco en sí constituyen una forma de publicidad indirecta. ${ }^{14,37}$ Este estudio demuestra que los empaques de los productos de tabaco son una forma de publicidad: hay variedad en cuanto a los tamaños, formas, colores y tipos de empaques, que en algunos casos resultan ser versiones coleccionables. Las advertencias sanitarias actuales sólo ocupan, en el mejor de los casos, $50 \%$ de la cara posterior, con la desventaja que el color del texto es igual al color de la cajetilla y el tamaño de la letra es mucho menor al de la etiqueta, por lo que no llama la atención y lo hace poco atractivo a la vista. Se ha comprobado que los mensajes de texto no comunican efectivamente el riesgo de las consecuencias del consumo y no incentivan la cesación. ${ }^{38}$ En este sentido, la LGCT, a través de la publicación de un acuerdo secretarial, ${ }^{39}$ introduce la mayoría de recomendaciones establecidas en el Artículo 11 del CMCT OMS y sus directrices. A partir del mes de septiembre de 2010, todos los empaques de los productos de tabaco que se vendan en México tendrán un pictograma o imagen impresa que estará colocada en 30\% de la cara anterior y una advertencia sanitaria impresa colocada en el $100 \%$ de la cara posterior y en una de las caras laterales; tendrán fondo negro con letras amarillas. Estos mensajes tendrán vigencia de un año y serán rotatorios. Se incorporan dos mensajes más: "Para venta exclusiva en México" y los datos de una línea telefónica de ayuda para dejar de fumar. De ninguna manera se permitirá que los mensajes den la falsa impresión que el producto es menos nocivo, por lo que no se observarán mensajes como "ligeros", "ultraligeros" "suaves". Por lo tanto, estará prohibida la colocación de calcomanías, sobres, cajas, fundas o cualquier otro artefacto que cubra las advertencias. México ha dado un paso importante en el control del tabaco al implementar esta estrategia; su desafío ahora es vigilar el estricto cumplimento de la Ley y las nuevas estrategias de la industria tabacalera para evadir las medidas de control establecidas.

En la actualidad, México no cuenta con un sistema de vigilancia de la epidemia del tabaquismo y sus estrategias de control que cumpla con todos los requerimientos establecidos en el CMCT OMS. En este manuscrito se describe por primera vez en México una metodología de vigilancia de las estrategias de la publicidad, promoción, venta, empaquetado y etiquetado de los productos de tabaco, acoplada al Sistema Global de Vigilancia Epidemiológica del Tabaco e implementada a través de un Sistema de Información Geográfica. La utilidad del SIG en el análisis espacial, la ubicación de los puntos de referencia (escuelas) y sus áreas de influencia, la identificación y caracterización de los puntos de venta y la posibilidad de localizar exactamente la posición de los espectaculares, 
lo convierte en una herramienta válida y confiable. ${ }^{16,37}$ Dado que esta herramienta utiliza una plataforma en internet, permite el registro de la información a nivel local y actualizaciones de manera periódica. Las mediciones no fueron realizadas en el mismo periodo, sin embargo partimos del supuesto de que durante este periodo no hubo cambios en el contexto que limitaran mostrar los resultados. Si se logra implementar esta estrategia, lo previsto es que las mediciones se realicen de manera simultánea. De igual manera, los indicadores de resultados se pueden analizar tanto a nivel local como a nivel nacional, constituyéndose una verdadera herramienta de vigilancia para los encargados de los programas de control del tabaco a nivel local como federal.

Con el fin de fortalecer y ampliar la cobertura del sistema de vigilancia es necesario considerar otros puntos de referencia, por ejemplo, incluir centros educativos de preparatoria y universitaria ubicados en las áreas urbanas, conurbadas y rurales, centros de recreación (parques, plazas comerciales, cines), así como unidades de atención médica (centros de salud y hospitales). Es importante establecer una estrategia para vigilar la realización de conciertos o eventos de filantropía patrocinados por la industria tabacalera o por terceros pagados por la industria. Durante febrero y marzo del 2008 se llevaron a cabo conciertos musicales (Marlboro MX Beat) patrocinados por la IT en las ciudades de Guadalajara, Puebla y Ciudad de México, dado que éstos no fueron realizados en el área de influencia de las escuelas no fueron registrados, tampoco denunciados ni sancionados, muy a pesar de que la ley los prohíbe completamente. Se plantea ampliar la búsqueda de promoción de estos eventos en medios de comunicación que son utilizados con mayor frecuencia por los jóvenes (por ejemplo internet), para dar seguimiento a esta actividad y sancionarla de acuerdo con lo establecido en la legislación.

\section{Conclusiones}

Alrededor de las escuelas existe una gran cantidad de puntos de venta que hacen accesibles y disponibles los productos de tabaco a los jóvenes. Los puestos de la calle hacen difícil el control ya que venden cigarros a menores y al menudeo a pesar de una ley que lo prohíbe. Urge un efectivo cumplimiento de la Ley y su Reglamento por parte de las autoridades sanitarias locales y federales.

Con un sistema de vigilancia se lograría contar con un continuo análisis, interpretación y retroalimentación sistemática de la información que ayude a identificar y caracterizar las estrategias de la industria y las medidas para el control del tabaco con varios propósitos; uno de ellos es poder tomar decisiones oportunas para prevenir el inicio del consumo en los jóvenes.
Las estrategias para el control de tabaco deben ser de cooperación interinstitucionales. La SSA, la SEP y las instituciones de la regulación sanitaria tendrán que trabajar coordinadamente para crear "escuelas 100\% libres de tabaco" y evalúen los contenidos de los textos escolares que abordan el problema en los adolescentes, ${ }^{40}$ además de que alrededor de las escuelas no se publicite, promocione o venda cigarros. Se debe buscar cumplir con la meta de prohibición total de la publicidad, promoción y patrocinio de los productos de tabaco, dictada en el CMCT OMS.

Dar a conocer a jóvenes, padres de familia, maestros y directores de escuelas la red de instituciones de ayuda para dejar de fumar, al igual que las líneas telefónicas gratuitas y los chats de internet disponibles las 24 horas para ayudar a los fumadores a abandonar el consumo de tabaco. Asimismo, es indispensable que se motive la denuncia ciudadana (01 800911 2000) por parte de la sociedad civil, ya que solamente así se podrá prevenir la iniciación y el consumo de tabaco en los jóvenes.

\section{Agradecimientos}

A las autoridades del Consejo Nacional contra las Adicciones y el personal de los Consejos Estatales contra las Adicciones de Baja California, Chihuahua, Jalisco, Morelos, Quintana Roo, Sinaloa, Tamaulipas y Zacatecas. Así como al personal de la Jurisdicción Sanitaria No. VII. Tapachula - Chiapas, a la Coordinación de Enfermedades Crónicas del Departamento de Medicina Preventiva de los Servicios de Salud de Puebla y al Instituto Mexiquense Contra las Adicciones del Estado de México. Al equipo colaborador del Centro de Información para Decisiones en Salud Pública del INSP, M en C. Juan Eugenio Hernández Ávila y la Lic. Ana Salgado Salgado, quienes diseñaron e implementaron el SIG. Un agradecimiento especial al Dr. Charles W. Warren y al equipo de la oficina sobre tabaquismo y salud del Centro para el Control y Prevención de Enfermedades (CDC por sus siglas en inglés).

Este proyecto fue financiado por Fogarty International Center HL-73699-05.

\section{Declaración de conflicto de intereses}

Declaramos no tener conflicto de intereses.

\section{Referencias}

1. Convenio Marco para el Control del Tabaco [consultado 2010 febrero 22]. Disponible en: http://www.who.int/fctc/es/.

2. Tobacco control tracking tools. General guide to using the surveillance tools. Johns Hopkins University. Bloomberg School of Public Health. Institute for Global Tobacco Control. 
3. Directrices para la aplicación del artículo II (empaquetado y etiquetado de los productos de tabaco) del Convenio Marco de la OMS para el control del Tabaco. Durban, Sudáfrica, 2008. [consultado 2010 febrero 22]. Disponible en: http://www.who.int/fctc/guidelines/article_II_es.pdf. 4. Directrices para la aplicación del artículo I3 (publicidad, promoción y patrocinio del tabaco) del Convenio Marco de la OMS para el control del tabaco. Durban, Sudáfrica, 2008. [consultado 2010 febrero 22]. Disponible en: http://www.who.int/fctc/guidelines/article_13_es.pdf. 5. Código Sanitario de los Estados Unidos Mexicanos. Publicado en el Diario Oficial de la Federación (DOF) el I 3 de junio de 1973. Disponible en: http://www.dof.gob.mx/nota to imagen.fs.php?cod.diario=197819\&pag $n a=0 \& f e c h a=13 / 03 / 1973$.

6. Ley General de Salud. México. Publicada en el Diario Oficial de la Federación el 7 de febrero de 1984 [consultado 2010 febrero 22]. Disponible en: http://www.diputados.gob.mx/LeyesBiblio/pdf//42.pdf 7. Artículo 210 de la Ley General de Salud. DOF 7 de febrero de 1984. 8. Artículos III7 y III8 del Reglamento de la Ley General de Salud en Materia de Control. Sanitario de Actividades, Establecimientos, Productos y Servicios, publicado en el DOF del 18 de enero de 1988.

9. Reforma a la Ley General de Salud, publicada en el DOF el I4 de junio de 1991.

10. Decreto que reforma la LGS, publicado en el DOF el 19 de enero de 2004.

II. Ley General para el Control del Tabaco. México. Publicada en el Diario Oficial de la Federación el 30 de mayo de 2008 [consultado 2010 febrero 22]. Disponible en: http://www.diputados.gob.mx/LeyesBiblio/ref//gct.htm. 12. Henriksen L, Feighery EC, Schleicher NC, Haladjian HH, Fortmann SP. Reaching youth at the point ofsale: Cigarette marketing is more prevalent in stores where adolescentsshop frequently. Tob Control 2004;13:3 I5-3/8. 13. DiFranza JR, Wellman RJ, Sargent JD Weitzman M, Hipple BJ,Winickoff JP.Tobacco Promotion and the Initiation of Tobacco Use:Assessing the Evidence for Causality. Pediatrics 2006; 1 17:1237-1248.

14. Loomis BR, Farrelly MC, Nonnemaker JM, Mann NHPoint of purchase cigarette promotions before and after the Master Settlement Agreement: Exploring retail scanner data. Tob Control 2006; |5, I40-142 .

15. Wakefield M, Germain D, Henriksen L. The effect of retail cigarette pack displays on impulse purchase. Addiction 2007; 103 (2): 322-328. 16. Hernández-Ávila JE,Tirado-Ramírez E, Santos-Luna R, ReynalesShigematsu LM. Use of GIS for billboards and points-of-sale surveillance in two Mexico cities. Salud Publica Mex 2007;49 suppl 2:S24I-S246.

17. Reynales-Shigematsu LM,Valdés-Salgado R, Rodríguez-Bolaños R, Lazcano-Ponce E, Hernández-Ávila M. Encuesta de Tabaquismo en Jóvenes en México.Análisis descriptivo 2003, 2005, 2006, 2008. Cuernavaca, Morelos: Instituto Nacional de Salud Pública, 2009.

18. Wade T, Sommer S.A to Z GIS,An illustrated dictionary of geographic information systems. 2a. edición. California: ESRI Press 2006:9.

19. Video.Vigilancia Epidemiológica de las estrategias de control de los productos del tabaco en términos de publicidad, mercadeo y venta, empaque y etiquetado. Instituto Nacional de Salud Pública. 2009. ISBN978607-7530-56-5 [consultado 2010 febrero 22]. Disponible en: http://www. insp.mx/tabaco/doars.php?idcola $=26$.

20. Malboro MXBeat. 2008 [consultado 2010 febrero 22]. Disponible en: http://consultaeventos.com/2008/0I/31/marlboro-beat-2008-mexico/ 21. World Health Organization (WHO). Report on the global Tobacco Epidemic 2008:The mpower package. Geneva:World Health Organization, 2008:36.

22. Andrews RL, Franke GR. The Determinants of Cigarette Consumption: A Meta-Analysis. Journal of Public Policy \& Marketing 1991;10:81-100. 23. Lovato C, Linn G, Stead LF, Best A. Impact of tobacco advertising and promotion on increasing adolescent smoking behaviors. Cohrane Database Syst Rev 2003;(4):CD003439.

24. Henriksen L, Feighery EC, Schleicher NC, Cowling DW, Kline RS, Fortman SP. Is adolescent smoking related to the density and proximity of tobacco outlets and retail cigarette advertising near schools? Prev Medic 2008;(47):210-214.

25. Lovato CY, Hsu HC, Sabiston CM, HaddV, Nykiforuk Cl.

Tobacco point-of-purchase marketing in school neighbourhoods and school smoking prevalence: a descriptive study. Can J Public Health 2007;(98):265-270.

26. Slater SJ, Chaloupka FJ,Wakefield M, Johnston LD, O'Malley PM. The Impact of Retail Cigarette Marketing Practices on Youth Smoking Uptake. Arch Pediatr Adolesc Med 2007; 16I (5):440-445

27. Sargent JD, Dalton M, Beach M. Exposure to cigarette promotions and smoking uptake in adolescents: evidence of a dose-response relation. Tobacco Control 2000;9(2):163-168.

28. Valdés-Salgado R,Thrasher J, Sánchez-Zamorano LM, Lazcano-Ponce E, Reynales-Shigematsu LM, Meneses-González F, et al. Los retos del convenio marco para el control del tabaco en México: un diagnóstico a partir de la Encuesta sobre Tabaquismo en Jóvenes. Salud Publica Mex 2006;48 supl I:5-16.

29. Kuri-Morales P, Cravioto P, Hoy M, Huerta S, Revuelta A, Jasso B et al. Illegal sales of cigarettes to minors-Mexico City, Mexico 1997. MMWR 1997;46:440-444

30. Tapia R, Kuri P, Cravioto P, Roldán JF, Hoy MJ.Venta de tabaco a menores de edad en Ciudad Juárez y el Distrito Federal. En: Primer informe sobre el combate al tabaquismo. México ante el Convenio Marco para el Control del Tabaco, México. Cuernavaca. Instituto Nacional de Salud Pública, 2005: 277-278.

3I. Adame-Moreno R, Ibarra O,Torres H, Kuri-Morales P, Hoy M, Tapia Conyer R et al. Illegal sales of cigarettes to minors-Ciudad Juárez, México; El Paso, Texas; and Las Cruces, New Mexico 1999. MMWR 1999;48:394-398. 32. Upadhyaya HP, Drobes DJ,Thomas SE. Reactivity to smoking cues in adolescent cigarette smokers. Addictive Behaviors 2004;29:849-885 33. Bordnick PS, Graap KM, Copp HL, Brooks J, Ferrer M.Virtual reality cue reactivity assessment in cigarette smokers. Cyberpsychol Behav 2005;8(5):487-492

34. Charlton A. Children and Smoking: the family circle. British Medical Bulletin 1996;52(I):90 - 107.

35. Vardavas C I, Connolly G N, Kafatos A G. Geographical information systems as a tool for monitoring tobacco industry advertising. Tob Control 2009;18:190-196.

36. Yong HH, Borland R, Hammond D, Sirirassamee B, Ritthiphakdee B, Awang $R$, et al. Levels and correlates of awareness of tobacco promotional activities among adult smokers in Malaysia and Thailand: findings from the International Tobacco Control Southeast Asia (ITC-SEA) Survey. Tob Control 2008; I7(I):46-52.

37. Wakefield M, Morley C, Horan JK, Cummings KM. The cigarette pack as image: new evidence from tobacco industry documents. Tob Control 2002; II;73-80.

38. Thrasher JF, Hammond D, Fong GT,Arillo-Santillán E. Smokers' reactions to cigarette package warnings with graphic imagery and with only text:A comparison between Mexico and Canada. Salud Publica Mex 2007;49 suppl 2:233-240.

39. Acuerdo publicado en el DOF el 24 de diciembre de 2009. [Consultado 2010 febrero 22]. Disponible en: http://www.dof.gob.mx/ nota_to_doc.php? codnota $=5126250$.

40. Willemsen MC, De ZwartWM. The effectiveness of policy and health education strategies for reducing adolescent smoking: a review of the evidence. J Adolesc 1999;22;587-599.

4I.Acuerdo publicado en el DOF el 24 de diciembre de 2009 42.Willemsen MC, De ZwartWM. The effectiveness of policy and health education strategies for reducing adolescent smoking: a review of the evidence. J Adolesc 1999;22;587-599. 